\title{
Fractional boundary value problems with Riemann-Liouville fractional derivatives
}

\author{
Jingjing Tan* and Caozong Cheng
}

"Correspondence:

tanjingjing1108@163.com

College of Applied Science, Beijing

University of Technology, Dawang

Road, Beijing, 100124, P.R. China

\begin{abstract}
In this paper, by employing two fixed point theorems of a sum operators, we investigate the existence and uniqueness of positive solutions for the following fractional boundary value problems: $-D_{0+}^{\alpha} x(t)=f(t, x(t), x(t))+g(t, x(t)), 0<t<1$, $1<\alpha<2$, where $D_{0+}^{\alpha}$ is the standard Riemann-Liouville fractional derivative, subject to either the boundary conditions $x(0)=x(1)=0$ or $x(0)=0, x(1)=\beta x(\eta)$ with $\eta, \beta \eta^{\alpha-1} \in(0,1)$. We also construct an iterative scheme to approximate the solution. As applications of the main results, two examples are given.
\end{abstract}

Keywords: fractional differential equation; boundary value problem; fixed point theorem; mixed monotone operators

\section{Introduction}

Fractional differential equations are important mathematical models of some practical problems in many fields such as polymer rheology, chemistry physics, heat conduction, fluid flows, electrical networks, and many other branches of science (see [1-7]). Consequently, the fractional calculus and its applications in various fields of science and engineering have received much attention, and many papers and books on fractional calculus, fractional differential equations have appeared (see [8-15]). It should be noted that most of the papers and books on fractional calculus are devoted to the solvability of the existence of positive solutions for nonlinear fractional differential equation boundary value problems (see [16-21]). However, there are few papers to deal with the existence and uniqueness of positive solutions for nonlinear fractional differential equation boundary value problems.

In this paper, we study the existence and uniqueness of positive solutions to the following two nonlinear Riemann-Liouville fractional differential equation boundary value problems:

$$
\left\{\begin{array}{l}
D_{0+}^{\alpha} x(t)=f(t, x(t), x(t))+g(t, x(t)), \quad 0<t<1,1<\alpha<2, \\
x(0)=x(1)=0,
\end{array}\right.
$$

and

$$
\left\{\begin{array}{l}
D_{0+}^{\alpha} x(t)=f(t, x(t), x(t))+g(t, x(t)), \quad 0<t<1,1<\alpha<2, \\
x(0)=0, \quad x(1)=\beta x(\eta),
\end{array}\right.
$$

where $D_{0+}^{\alpha}$ is the standard Riemann-Liouville fractional derivative and $\eta, \beta \eta^{\alpha-1} \in(0,1)$.

\section{Springer}

(c) 2015 Tan and Cheng: licensee Springer. This is an Open Access article distributed under the terms of the Creative Commons Attribution License (http://creativecommons.org/licenses/by/4.0), which permits unrestricted use, distribution, and reproduction in any medium, provided the original work is properly credited. 
In [22], by means of the Krasnoselskii fixed point theorem, Jiang and Yuan obtained the existence of positive solutions to the following nonlinear fractional differential equation Dirichlet-type boundary value problem:

$$
\left\{\begin{array}{l}
D_{0+}^{\alpha} u(t)+f(t, u(t))=0, \quad 0<t<1,1<\alpha \leq 2 \\
u(0)=u(1)=0
\end{array}\right.
$$

Bai and Lü [23] obtained some existence results of positive solutions of the problem by (3) using some fixed point theorems on cone. A more general equation with $f$ depending on derivatives was studied in [24]. In [25], the authors studied the boundary value problems of the fractional order differential equation:

$$
\left\{\begin{array}{l}
D_{0+}^{\alpha} u(t)=f(t, u(t)), \quad 0<t<1,1<\alpha<2, \\
u(0)=0, \quad u(1)=\beta u(\eta),
\end{array}\right.
$$

where $0 \leq \beta, \eta \leq 1$. They established some existence results of positive solutions. In [26], the boundary condition $D_{0_{+}}^{\beta} u(1)=a D_{0+}^{\beta} u(\xi)$ was considered, where $0 \leq \beta \leq 1,0 \leq a \leq 1$, $0<\xi<1$. They obtained the multiple positive solutions by the Leray-Schauder nonlinear alternative and the fixed point theorem on cones.

Motivated by the results mentioned above, in this paper, we study the existence and uniqueness of positive solutions for the BVP (1) and (2). We have found that no result has been established for the existence and uniqueness of positive solutions for the problem (1) and (2) of fractional differential equation. This paper aims to establish the existence and uniqueness of positive solutions for the problem (1) and (2). The technique relies on two fixed point theorems of a sum operator. The method used in this paper is different from the ones in the papers mentioned above. In addition, we also construct an iterative sequence to approximate the solution.

The rest of this paper is organized as follows. In Section 2, we recall some definitions and facts. In Section 3, the main results are discussed by using the properties of the Green function and the fixed point theorem on mixed monotone operators. Finally, in Section 4, we give two examples to demonstrate our results.

\section{Preliminaries}

In this section, we recall some definitions and facts which will be used in the later analysis. For convenience, we introduce the following notations:

(1) $\lceil\alpha\rceil$ : the smallest integer greater than or equal to $\alpha$;

(2) $[\alpha]$ : the integer part of the number $\alpha$;

(3) $\Gamma(\alpha)=\int_{0}^{\infty} t^{\alpha-1} e^{-t} d t$.

Definition 2.1 ([27]) The Riemann-Liouville fractional integral of order $\alpha>0$ of a function $f:(0, \infty) \rightarrow R$ is given by

$$
I_{0+}^{\alpha} f(x)=\frac{1}{\Gamma(\alpha)} \int_{0}^{x} \frac{f(t)}{(x-t)^{1-\alpha}} d t
$$

provided that the right side is pointwise defined on $(0, \infty)$. 
Definition 2.2 ([27]) The Riemann-Liouville fractional derivative of order $\alpha>0$ of a function $f:(0, \infty) \rightarrow R$ is given by

$$
D_{0+}^{\alpha} f(x)=\frac{1}{\Gamma(n-\alpha)}\left(\frac{d}{d x}\right)^{n} \int_{0}^{x} \frac{f(t)}{(x-t)^{\alpha-n-1}} d t
$$

where $n=[\alpha]+1$, provided that the right side is pointwise defined on $(0, \infty)$.

Lemma 2.1 ([23]) Assume that $u \in C(0,1) \cap L(0,1)$ with a fractional derivative of order $\alpha>0$. Then

$$
I_{0+}^{\alpha} D_{0+}^{\alpha} u(t)=u(t)+c_{1} t^{\alpha-1}+c_{2} t^{\alpha-2}+\cdots+c_{N} t^{\alpha-N}
$$

for some $c_{i} \in R, i=1,2,3, \ldots, N$, where $N=\lceil\alpha\rceil$.

Denote by $E$ a real Banach space. Recall that a non-empty closed convex set $P \subset E$ is a cone if it satisfies (i) $x \in P, \lambda \geq 0 \Rightarrow \lambda x \in P$ and (ii) $x \in P,-x \in P \Rightarrow x=\theta$. Suppose that $(E,\|\cdot\|)$ is a real Banach space which is partially ordered by a cone $P \subset E$, i.e., $x \leq y$ if and only if $y-x \in P$. The cone $P$ is called normal if there exists a constant $N>0$ such that, for all $x, y \in E, \theta \leq x \leq y$ implies $\|x\| \leq N\|y\|$, and $N$ is called the normal constant. Putting $P^{o}=\{x \in P \mid x$ is an interior point of $P\}$, the cone $P$ is said to be solid if its interior $P^{o}$ is non-empty. If $x_{1}, x_{2} \in E$, the set $\left[x_{1}, x_{2}\right]=\left\{x \in E \mid x_{1} \leq x \leq x_{2}\right\}$ is called the order interval between $x_{1}$ and $x_{2}$. We say that an operator $A: E \rightarrow E$ is increasing (decreasing) if $x \leq y$ implies $A x \leq A y(A x \geq A y)$.

For $x, y \in E$, the notation $x \sim y$ means that there exist $\lambda>0$ and $\mu>0$ such that $\lambda x \leq y \leq$ $\mu x$. Clearly, $\sim$ is an equivalence relation. Given $h>\theta$ (i.e., $h \geq \theta$ and $h \neq \theta$ ), let $P_{h}=\{x \in$ $E \mid x \sim h\}$. If $P_{h} \subset P^{o}$, then $P_{h}=P^{o}$.

The basic space used in this paper is the space $C[0,1]$, it is a Banach space if it is endowed with the norm $\|x\|=\sup \{|x(t)|: t \in[0,1]\}$ for any $x \in C[0,1]$. Notice that this space can be equipped with a partial order given by $x, y \in C[0,1], x \leq y \Longleftrightarrow x(t) \leq y(t)$ for $t \in[0,1]$. Let $P \subset C[0,1]$ by $P=\{x \in C[0,1] \mid x(t) \geq 0, t \in[0,1]\}$. Clearly $P$ is a normal cone in $C[0,1]$ and the normality constant is 1 .

Lemma 2.2 ([22]) Given $y \in C[0,1]$ and $1<\alpha \leq 2$, the unique solution of

$$
\left\{\begin{array}{l}
D_{0+}^{\alpha} u(t)+y(t)=0, \quad 0<t<1, \\
u(0)=u(1)=0
\end{array}\right.
$$

is

$$
u(t)=\int_{0}^{1} G(t, s) y(s) d s
$$

where $G(t, s)$ is the Green function of the $B V P(4)$ and is given by

$$
G(t, s)= \begin{cases}\frac{[t(1-s)]^{\alpha-1}-(t-s)^{\alpha-1}}{\Gamma(\alpha)}, & s \leq t, \\ \frac{[t(1-s)]^{\alpha-1}}{\Gamma(\alpha)}, & t \leq s .\end{cases}
$$


Lemma 2.3 ([22]) $G(t, s)$ satisfies the following properties:

(1) $G(t, s) \in C([0,1] \times[0,1])$;

(2) $G(t, s)>0$ for $t, s \in(0,1)$;

(3) for all $t, s \in(0,1)$,

$$
\frac{\alpha-1}{\Gamma(\alpha)} t^{\alpha-1}(1-t)(1-s)^{\alpha-1} s \leq G(t, s) \leq \frac{1}{\Gamma(\alpha)} t^{\alpha-1}(1-t)(1-s)^{\alpha-2} .
$$

Lemma 2.4 Assume that $f:[0,1] \times[0, \infty) \times[0, \infty) \rightarrow[0, \infty)$ and $g:[0,1] \times[0, \infty) \rightarrow$ $[0, \infty)$ are continuous. A function $u \in P$ is a solution of the BVP (1) if and only if it is a solution of the integral equation

$$
u(t)=\int_{0}^{1} G(t, s)[f(s, u(s), u(s))+g(s, u(s))] d s .
$$

Proof The proof is similar to Lemma 2.2 and omitted.

Lemma 2.5 ([25]) Given $y \in C[0,1]$, the problem

$$
\left\{\begin{array}{l}
D_{0+}^{\alpha} u(t)+y(t)=0, \quad 0<t<1 \\
u(0)=0, \quad u(1)=\beta u(\eta)
\end{array}\right.
$$

is equivalent to

$$
u(t)=\int_{0}^{1} H(t, s) y(s) d s,
$$

where $H(t, s)$ is the Green function of the BVP (7) and is given by

$$
H(t, s)= \begin{cases}\frac{[t(1-s)]^{\alpha-1}-\beta t^{\alpha-1}(\eta-s) \alpha-1}{\left.\left.\left(1-\beta \eta^{\alpha-1}\right)(t-s)\right)^{\alpha-1}(1-\beta \eta)\right)^{\alpha-1}}, & 0 \leq s \leq t \leq 1, s \leq \eta, \\ \frac{[t(1-s)]^{\alpha-1}-(t-s)^{\alpha-1}\left(1-\beta \eta^{\alpha-1}\right)}{\left(1-\beta \eta^{\alpha-1}\right) \Gamma(\alpha)}, & 0 \leq \eta \leq s \leq t \leq 1, \\ \frac{[t(1-s)]^{\alpha-1}-\beta t^{\alpha-1}(\eta-s)^{\alpha-1}}{\left(1-\beta \beta^{\alpha-1}\right) \Gamma(\alpha)}, & 0 \leq t \leq s \leq \eta \leq 1, \\ \frac{[t(1-s))^{\alpha-1}}{\left(1-\beta \eta^{\alpha-1}\right) \Gamma(\alpha)}, & 0 \leq t \leq s \leq 1, \eta \leq s .\end{cases}
$$

Lemma 2.6 ([25]) $H(t, s)$ has the following properties:

(1) $H(t, s) \in C([0,1] \times[0,1])$;

(2) $H(t, s)>0$ for $t, s \in(0,1)$;

(3) for all $t, s \in(0,1)$,

$$
\frac{M_{0} t^{\alpha-1} s(1-s)^{\alpha-1}}{\Gamma(\alpha)\left(1-\beta \eta^{\alpha-1}\right)} \leq H(t, s) \leq \frac{t^{\alpha-1}(1-s)^{\alpha-1}}{\Gamma(\alpha)\left(1-\beta \eta^{\alpha-1}\right)},
$$

where $0<M_{0}=\min \left\{1-\beta \eta^{\alpha-1}, \beta \eta^{\alpha-1}\right\}<1$.

Lemma 2.7 Assume that $f:[0,1] \times[0, \infty) \times[0, \infty) \rightarrow[0, \infty)$ and $g:[0,1] \times[0, \infty) \rightarrow$ $[0, \infty)$ are continuous. A function $u \in P$ is a solution of the BVP (2) if and only if it is a solution of the integral equation

$$
u(t)=\int_{0}^{1} H(t, s)[f(s, u(s), u(s))+g(s, u(s))] d s .
$$


Proof The proof is similar to Lemma 2.5 and omitted.

Definition 2.3 ([28]) $A: P \times P \rightarrow P$ is said to be a mixed monotone operator if $A(u, v)$ is increasing in $u$ and decreasing in $v$, i.e., $u_{i}, v_{i}(i=1,2) \in P, u_{1} \leq u_{2}, v_{1} \geq v_{2}$ imply $A\left(u_{1}, v_{1}\right) \leq$ $A\left(u_{2}, v_{2}\right)$. The element $x \in P$ is called a fixed point of $A$ if $A(x, x)=x$.

Definition 2.4 ([29]) An operator $A: E \rightarrow E$ is said to be positive homogeneous if it satisfies $A(t x)=t A x, \forall t>0, x \in E$. An operator $A: P \rightarrow P$ is said to be sub-homogeneous if it satisfies

$$
A(t x) \geq t A x, \quad \forall t \in(0,1), x \in P .
$$

Definition 2.5 ([29]) Let $D=P$ and $r$ be a real number with $0 \leq r<1$. An operator $A$ : $P \rightarrow P$ is said to be $r$-concave if it satisfies

$$
A(t x) \geq t^{r} A x, \quad \forall t \in(0,1), x \in D .
$$

Lemma 2.8 ([28]) Let $h>\theta$ and $\beta \in(0,1) . A: P \times P \rightarrow P$ is a mixed monotone operator and satisfies

$$
A\left(t x, t^{-1} y\right) \geq t^{\beta} A(x, y), \quad \forall t \in(0,1), x, y \in P .
$$

$B: P \rightarrow P$ is an increasing sub-homogeneous operator. Assume that

(i) there is a $h_{0} \in P_{h}$ such that $A\left(h_{0}, h_{0}\right) \in P_{h}$ and $B h_{0} \in P_{h}$;

(ii) there exists a constant $\delta_{0}>0$ such that $A(x, y) \geq \delta_{0} B x, \forall x, y \in P$.

Then

(1) $A: P_{h} \times P_{h} \rightarrow P_{h}, B: P_{h} \rightarrow P_{h}$;

(2) there exist $u_{0}, v_{0} \in P_{h}$ and $\gamma \in(0,1)$ such that $\gamma v_{0} \leq u_{0}<v_{0}$, $u_{0} \leq A\left(u_{0}, v_{0}\right)+B u_{0} \leq A\left(v_{0}, u_{0}\right)+B v_{0} \leq v_{0}$

(3) the operator equation $A(x, x)+B x=x$ has a unique solution $x^{*}$ in $P_{h}$;

(4) for any initial values $x_{0}, y_{0} \in P_{h}$, constructing successively sequences $x_{n}=A\left(x_{n-1}, y_{n-1}\right)+B x_{n-1}, y_{n}=A\left(y_{n-1}, x_{n-1}\right)+B y_{n-1}, n=1,2, \ldots$, we have $x_{n} \rightarrow x^{*}$ and $y_{n} \rightarrow x^{*}$ as $n \rightarrow \infty$.

Lemma 2.9 ([28]) Let $h>\theta$ and $\alpha \in(0,1) . A: P \times P \rightarrow P$ is a mixed monotone operator and satisfies

$$
A\left(t x, t^{-1} y\right) \geq t A(x, y), \quad \forall t \in(0,1), x, y \in P .
$$

$B: P \rightarrow P$ is an increasing $\alpha$-concave operator. Assume that

(i) there is a $h_{0} \in P_{h}$ such that $A\left(h_{0}, h_{0}\right) \in P_{h}$ and $B h_{0} \in P_{h}$;

(ii) there exists a constant $\delta_{0}>0$ such that $A(x, y) \leq \delta_{0} B x, \forall x, y \in P$.

Then

(1) $A: P_{h} \times P_{h} \rightarrow P_{h}$ and $B: P_{h} \rightarrow P_{h}$;

(2) there exist $u_{0}, v_{0} \in P_{h}$ and $\gamma \in(0,1)$ such that $\gamma v_{0} \leq u_{0}<v_{0}$, $u_{0} \leq A\left(u_{0}, v_{0}\right)+B u_{0} \leq A\left(v_{0}, u_{0}\right)+B v_{0} \leq v_{0} ;$

(3) the operator equation $A(x, x)+B x=x$ has a unique solution $x^{*}$ in $P_{h}$; 
(4) for any initial values $x_{0}, y_{0} \in P_{h}$, constructing successively the sequences

$x_{n}=A\left(x_{n-1}, y_{n-1}\right)+B x_{n-1}, y_{n}=A\left(y_{n-1}, x_{n-1}\right)+B y_{n-1}, n=1,2, \ldots$, we have $x_{n} \rightarrow x^{*}$ and $y_{n} \rightarrow x^{*}$ as $n \rightarrow \infty$.

Lemma 2.10 ([28]) Let $\alpha \in(0,1)$ and $A: P \times P \rightarrow P$ be a mixed monotone operator. Assume that (12) holds and there is $h_{0}>\theta$ such that $A\left(h_{0}, h_{0}\right) \in P_{h}$.

Then

(1) $A: P_{h} \times P_{h} \rightarrow P_{h}$;

(2) there exist $u_{0}, v_{0} \in P_{h}$ and $r \in(0,1)$ such that $r v_{0} \leq u_{0} \leq v_{0}$, $u_{0} \leq A\left(u_{0}, v_{0}\right) \leq A\left(v_{0}, u_{0}\right) \leq v_{0}$;

(3) the operator equation $A(x, x)=x$ has a unique solution $x^{*}$ in $P_{h}$;

(4) for any initial values $x_{0}, y_{0} \in P_{h}$, constructing successively the sequences $x_{n}=A\left(x_{n-1}, y_{n-1}\right), y_{n}=A\left(y_{n-1}, x_{n-1}\right), n=1,2, \ldots$, we have $x_{n} \rightarrow x^{*}$ and $y_{n} \rightarrow x^{*}$ as $n \rightarrow \infty$.

\section{Main results}

In this section, we establish the existence and uniqueness of positive solutions results for the problems (1) and (2), respectively.

First, we give the existence and uniqueness of positive solutions to the problem (1).

\section{Theorem 3.1 Assume that}

$\left(\mathrm{H}_{1}\right) f(t, x, y):[0,1] \times[0,+\infty) \times[0,+\infty) \rightarrow[0,+\infty)$ is continuous and increasing in $x \in$ $[0,+\infty)$ for fixed $t \in[0,1]$ and $y \in[0,+\infty)$ decreasing in $y \in[0,+\infty)$ for fixed $t \in[0,1]$ and $x \in[0,+\infty)$;

$\left(\mathrm{H}_{2}\right) g(t, x):[0,1] \times[0,+\infty) \rightarrow[0,+\infty)$ is continuous and increasing in $x \in[0,+\infty)$ for fixed $t \in[0,1], g(t, 0) \not \equiv 0$;

$\left(\mathrm{H}_{3}\right)$ there exists a constant $\delta_{0}>0$ such that $f(t, x, y) \geq \delta_{0} g(t, x), t \in[0,1], x, y \geq 0$;

$\left(\mathrm{H}_{4}\right) g(t, \lambda x) \geq \lambda g(t, x)$ for $\lambda \in(0,1), t \in[0,1], u \in[0,+\infty)$ and there exists a constant $\xi \in$ $(0,1)$ such that $f\left(t, \lambda x, \lambda^{-1} y\right) \geq \lambda^{\xi} f(t, x, y), \forall t \in[0,1], x, y \in[0,+\infty)$.

Then

(1) there exist $x_{0}, y_{0} \in P_{h}$ and $\gamma \in(0,1)$ such that $\gamma y_{0} \leq x_{0}<y_{0}$ and

$$
\begin{array}{ll}
x_{0}(t) \leq \int_{0}^{1} G(t, s)\left[f\left(t, x_{0}(s), y_{0}(s)\right)+g\left(s, x_{0}(s)\right)\right] d s, & t \in[0,1], \\
y_{0}(t) \geq \int_{0}^{1} G(t, s)\left[f\left(s, x_{0}(s), y_{0}(s)\right)+g\left(s, x_{0}(s)\right)\right] d s, & t \in[0,1],
\end{array}
$$

where $h(t)=t^{\alpha-1}(1-t), t \in[0,1]$;

(2) the BVP (1) has a unique positive solution $x^{*}$ in $P_{h}$. For any $x_{0}, y_{0} \in P_{h}$, constructing successively the sequences

$$
\begin{array}{ll}
x_{n+1}(t)=\int_{0}^{1} G(t, s)\left[f\left(s, x_{n}(s), y_{n}(s)\right)+g\left(s, x_{n}(s)\right)\right] d s, & n=0,1,2, \ldots, \\
y_{n+1}(t)=\int_{0}^{1} G(t, s)\left[f\left(s, y_{n}(s), x_{n}(s)\right)+g\left(s, y_{n}(s)\right)\right] d s, & n=0,1,2, \ldots,
\end{array}
$$

we have $\left\|x_{n}-x^{*}\right\| \rightarrow 0$ and $\left\|y_{n}-x^{*}\right\| \rightarrow 0$ as $n \rightarrow \infty$. 
Proof Firstly, according to Lemma 2.4, the BVP (1) is equivalent to the integral formulation given by

$$
x(t)=\int_{0}^{1} G(t, s)[f(s, x(s), x(s))+g(s, x(s))] d s .
$$

Let $A_{1}: P \times P \rightarrow E$ be the operator defined by

$$
A_{1}(x, y)(t)=\int_{0}^{1} G(t, s) f(s, x(s), y(s)) d s
$$

and $B_{1}: P \rightarrow E$ be the operator defined by

$$
\left(B_{1} x\right)(t)=\int_{0}^{1} G(t, s) g(s, x(s)) d s
$$

It is simple to show that $x$ is the solution of the BVP (1) if and only if $x$ solves the operator equation $x=A_{1}(x, x)+B_{1} x$. From $\left(\mathrm{H}_{1}\right)$ and $\left(\mathrm{H}_{2}\right)$ we know that $A_{1}: P \times P \rightarrow P$ and $B_{1}: P \rightarrow P$.

Secondly, we show that $A_{1}$ and $B_{1}$ satisfy all the assumptions of Lemma 2.8 . To begin with, we prove that $A_{1}$ is a mixed monotones operator. In fact, for $x_{i}, y_{i} \in P, i=1,2$ with $x_{1} \geq x_{2}, y_{1} \leq y_{2}$, we know that $x_{1}(t) \geq x_{2}(t)$ and $y_{1}(t) \leq y_{2}(t)$ for all $t \in[0,1]$. From $\left(\mathrm{H}_{1}\right)$ and Lemma 2.3 we know that $A_{1}\left(x_{1}, y_{1}\right)(t)=\int_{0}^{1} G(t, s) f\left(s, x_{1}(s), y_{1}(s)\right) d s \geq$ $\int_{0}^{1} G(t, s) f\left(s, x_{2}(s), y_{2}(s)\right) d s=A_{1}\left(x_{2}, y_{2}\right)(t)$. That is, $A_{1}\left(x_{1}, y_{1}\right) \geq A_{1}\left(x_{2}, y_{2}\right)$. Furthermore, it follows from $\left(\mathrm{H}_{2}\right)$ and Lemma 2.3 that $B_{1}$ is increasing. Next we prove that $A_{1}$ satisfies (12). For any $\lambda \in(0,1)$ and $x, y \in P$, together with $\left(\mathrm{H}_{4}\right)$, we have

$$
\begin{aligned}
A_{1}\left(\lambda x, \lambda^{-1} y\right)(t) & =\int_{0}^{1} G(t, s) f\left(s, \lambda x(s), \lambda^{-1} y(s)\right) d s \\
& \geq \lambda^{\xi} \int_{0}^{1} G(t, s) f(s, x(s), y(s)) d s \\
& =\lambda^{\xi} A_{1}(x, y)(t) .
\end{aligned}
$$

That is, $A_{1}\left(\lambda x, \lambda^{-1} y\right) \geq \lambda^{\xi} A_{1}(x, y)$ for $\lambda \in(0,1), x, y \in P$. Hence the operator $A_{1}$ satisfies (12). Also, for any $\lambda \in(0,1), x \in P$, using $\left(\mathrm{H}_{4}\right)$ we have

$$
B_{1}(\lambda x)(t)=\int_{0}^{1} G(t, s) g(s, \lambda x(s)) d s \geq \lambda \int_{0}^{1} G(t, s) g(s, x(s)) d s=\lambda B_{1} x(t) .
$$

Thus, $B_{1}(\lambda x) \geq \lambda B_{1}(x)$ for any $\lambda \in(0,1), x \in P$. Hence the operator $B_{1}$ is sub-homogeneous. Now we prove that $A_{1}(h, h) \in P_{h}$ and $B_{1} h \in P_{h}$. We only need to verify the following conclusions:

(a) $\exists a_{1}>0$ and $a_{2}>0$, such that, for all $t \in[0,1], a_{2} h(t) \leq A_{1}(h, h)(t) \leq a_{1} h(t)$;

(b) $\exists b_{1}>0$ and $b_{2}>0$, such that, for all $t \in[0,1], b_{2} h(t) \leq B_{1} h(t) \leq b_{1} h(t)$.

Let

$$
a_{1}:=\frac{1}{\Gamma(\alpha)} \int_{0}^{1}(1-s)^{\alpha-2} f(s, 1,0) d s
$$




$$
\begin{aligned}
a_{2} & :=\frac{\alpha-1}{\Gamma(\alpha)} \int_{0}^{1}(1-s)^{\alpha-2} s f(s, 0,1) d s, \\
b_{1} & :=\frac{1}{\Gamma(\alpha)} \int_{0}^{1}(1-s)^{\alpha-2} g(s, 1) d s \\
b_{2} & :=\frac{\alpha-1}{\Gamma(\alpha)} \int_{0}^{1}(1-s)^{\alpha-2} s g(s, 0) d s .
\end{aligned}
$$

It follows from $\left(\mathrm{H}_{1}\right),\left(\mathrm{H}_{2}\right)$, and Lemma 2.3 that, for any $t \in[0,1]$,

$$
\begin{aligned}
A_{1}(h, h)(t) & =\int_{0}^{1} G(t, s) f(s, h(s), h(s)) d s \\
& \leq \frac{1}{\Gamma(\alpha)} h(t) \int_{0}^{1}(1-s)^{\alpha-2} f(s, 1,0) d s \\
& =a_{1} h(t)
\end{aligned}
$$

and

$$
\begin{aligned}
A_{1}(h, h)(t) & =\int_{0}^{1} G(t, s) f(s, h(s), h(s)) d s \\
& \geq \frac{\alpha-1}{\Gamma(\alpha)} h(t) \int_{0}^{1}(1-s)^{\alpha-1} f(s, 0,1) d s \\
& =a_{2} h(t) .
\end{aligned}
$$

According to $\left(\mathrm{H}_{1}\right),\left(\mathrm{H}_{2}\right)$, and $\left(\mathrm{H}_{3}\right)$, we get

$$
f(s, 1,0) \geq f(s, 0,1) \geq \delta_{0} g(s, 0) \geq 0 .
$$

Since $g(t, 0) \not \equiv 0$, we obtain

$$
\int_{0}^{1} f(s, 1,0) d s \geq \int_{0}^{1} f(s, 0,1) d s \geq \delta_{0} \int_{0}^{1} g(s, 0) d s>0
$$

and, in consequence, $a_{1}>0$ and $a_{2}>0$. Thus, $a_{2} h(t) \leq A_{1}(h, h)(t) \leq a_{1} h(t), t \in[0,1]$, and hence we get (a). In the same way, we get

$$
\begin{aligned}
& B_{1} h(t) \leq \frac{1}{\Gamma(\alpha)} h(t) \int_{0}^{1}(1-s)^{\alpha-2} g(s, 1) d s=b_{1} h(t), \\
& B_{1} h(t) \geq \frac{\alpha-1}{\Gamma(\alpha)} h(t) \int_{0}^{1}(1-s)^{\alpha-2} s g(s, 0) d s=b_{2} h(t) .
\end{aligned}
$$

From $g(t, 0) \not \equiv 0$ we have $b_{1}>0$ and $b_{2}>0$. Therefore, $b_{2} h(t) \leq B_{1} h(t) \leq b_{1} h(t), t \in[0,1]$. Thus, (b) is satisfied. Hence the condition (i) of Lemma 2.8 is proved. For $x, y \in P$, and for any $t \in[0,1]$, from $\left(\mathrm{H}_{3}\right)$ we have

$$
A_{1}(x, y)(t)=\int_{0}^{1} G(t, s) f(s, x(s), y(s)) d s \geq \delta_{0} \int_{0}^{1} G(t, s) g(s, x(s)) d s=\delta_{0} B_{1} x(t)
$$


that is, $A_{1}(x, y) \geq \delta_{0} B_{1} x, \forall x, y \in P$. It follows from the above inequality that the condition (ii) of Lemma 2.8 is satisfied. Applying Lemma 2.8, we can get the conclusion of Theorem 3.1.

Combining the proof of Theorem 3.1 with Lemma 2.10, we can prove the following conclusion.

\section{Corollary 3.1 Assume that}

$\left(\mathrm{H}_{1}\right)^{\prime} f(t, x, y):[0,1] \times[0,+\infty) \times[0,+\infty) \rightarrow[0,+\infty)$ is continuous and increasing in $x \in$ $[0,+\infty)$ for fixed $t \in[0,1]$ and $y \in[0,+\infty)$ decreasing in $y \in[0,+\infty)$ for fixed $t \in[0,1]$ and $x \in[0,+\infty), f(t, 0,1) \not \equiv 0$;

$\left(\mathrm{H}_{2}\right)^{\prime}$ there exists a constant $\xi \in(0,1)$ such that $f\left(t, \lambda x, \lambda^{-1} y\right) \geq \lambda^{\xi} f(t, x, y), t \in[0,1], \lambda \in$ $(0,1), x, y \in[0,+\infty)$.

\section{Then}

(1) there exist $x_{0}, y_{0} \in P_{h}$ and $\gamma \in(0,1)$ such that $\gamma y_{0} \leq x_{0}<y_{0}$ and

$$
\begin{aligned}
& x_{0}(t) \leq \int_{0}^{1} G(t, s) f\left(t, x_{0}(s), y_{0}(s)\right) d s, \quad t \in[0,1], \\
& y_{0}(t) \geq \int_{0}^{1} G(t, s) f\left(s, y_{0}(s), x_{0}(s)\right) d s, \quad t \in[0,1]
\end{aligned}
$$

where $h(t)=t^{\alpha-1}(1-t), t \in[0,1]$;

(2) the problem

$$
\left\{\begin{array}{l}
D_{0+}^{\alpha} x(t)=f(t, x(t), x(t)), \quad 0<t<1 \\
x(0)=x(1)=0
\end{array}\right.
$$

has a unique positive solution $x^{*}$ in $P_{h}$ and for any $x_{0}, y_{0} \in P_{h}$, constructing successively the sequences

$$
\begin{array}{ll}
x_{n+1}(t)=\int_{0}^{1} G(t, s) f\left(s, x_{n}(s), y_{n}(s)\right) d s, & n=0,1,2, \ldots, \\
y_{n+1}(t)=\int_{0}^{1} G(t, s) f\left(s, y_{n}(s), x_{n}(s)\right) d s, & n=0,1,2, \ldots,
\end{array}
$$

we have $\left\|x_{n}-x^{*}\right\| \rightarrow 0$ and $\left\|y_{n}-x^{*}\right\| \rightarrow 0$ as $n \rightarrow \infty$.

By using Lemma 2.9 we can also prove the following theorem.

Theorem 3.2 Assume that $\left(\mathrm{H}_{1}\right),\left(\mathrm{H}_{2}\right)$, and

$\left(\mathrm{H}_{5}\right)$ there exists a constant $\delta_{0}>0$ such that $f(t, x, y) \leq \delta_{0} g(t, x), t \in[0,1], x, y \geq 0$;

$\left(\mathrm{H}_{6}\right)$ there exists a constant $\xi \in(0,1)$ such that $g(t, \lambda x) \geq \lambda^{\xi} g(t, x)$ for $\lambda \in(0,1), t \in[0,1]$, $x \in[0,+\infty)$, and $f\left(t, \lambda x, \lambda^{-1} y\right) \geq \lambda f(t, x, y), \forall t \in[0,1], \lambda \in(0,1), x, y \in[0,+\infty)$. 
Then

(1) there exist $x_{0}, y_{0} \in P_{h}$ and $\gamma \in(0,1)$ such that $\gamma y_{0} \leq x_{0}<y_{0}$ and

$$
\begin{array}{ll}
x_{0}(t) \leq \int_{0}^{1} G(t, s)\left[f\left(t, x_{0}(s), y_{0}(s)\right)+g\left(s, x_{0}(s)\right)\right] d s, & t \in[0,1], \\
y_{0}(t) \geq \int_{0}^{1} G(t, s)\left[f\left(s, y_{0}(s), x_{0}(s)\right)+g\left(s, x_{0}(s)\right)\right] d s, & t \in[0,1],
\end{array}
$$

where $h(t)=t^{\alpha-1}(1-t), t \in[0,1]$;

(2) the BVP (1) has a unique positive solution $x^{*}$ in $P_{h}$ and for any $x_{0}, y_{0} \in P_{h}$, constructing successively the sequences

$$
\begin{array}{ll}
x_{n+1}(t)=\int_{0}^{1} G(t, s)\left[f\left(s, x_{n}(s), y_{n}(s)\right)+g\left(s, x_{n}(s)\right)\right] d s, & n=0,1,2, \ldots, \\
y_{n+1}(t)=\int_{0}^{1} G(t, s)\left[f\left(s, y_{n}(s), x_{n}(s)\right)+g\left(s, y_{n}(s)\right)\right] d s, & n=0,1,2, \ldots,
\end{array}
$$

we have $\left\|x_{n}-x^{*}\right\| \rightarrow 0$ and $\left\|y_{n}-x^{*}\right\| \rightarrow 0$ as $n \rightarrow \infty$.

Proof The proof is similar to that given for Theorem 3.1. We omit it.

Next, we present the existence and uniqueness of a positive solution to the problem (2).

Theorem 3.3 Assume that $\left(\mathrm{H}_{1}\right)-\left(\mathrm{H}_{4}\right)$ hold. Then

(1) there exist $x_{0}, y_{0} \in P_{h}$ and $\gamma \in(0,1)$ such that $\gamma y_{0} \leq x_{0}<y_{0}$ and

$$
\begin{aligned}
& x_{0}(t) \leq \int_{0}^{1} H(t, s)\left[f\left(t, x_{0}(s), y_{0}(s)\right)+g\left(s, x_{0}(s)\right)\right] d s, \quad t \in[0,1], \\
& y_{0}(t) \geq \int_{0}^{1} H(t, s)\left[f\left(s, y_{0}(s), x_{0}(s)\right)+g\left(s, x_{0}(s)\right)\right] d s, \quad t \in[0,1],
\end{aligned}
$$

where $h(t)=t^{\alpha-1}, t \in[0,1]$;

(2) the BVP (2) has a unique positive solution $x^{*}$ in $P_{h}$ and for any $x_{0}, y_{0} \in P_{h}$, constructing successively the sequences

$$
\begin{aligned}
& x_{n+1}(t)=\int_{0}^{1} H(t, s)\left[f\left(s, x_{n}(s), y_{n}(s)\right)+g\left(s, x_{n}(s)\right)\right] d s, \quad n=0,1,2, \ldots, \\
& y_{n+1}(t)=\int_{0}^{1} H(t, s)\left[f\left(s, y_{n}(s), x_{n}(s)\right)+g\left(s, y_{n}(s)\right)\right] d s, \quad n=0,1,2, \ldots
\end{aligned}
$$

we have $\left\|x_{n}-x^{*}\right\| \rightarrow 0$ and $\left\|y_{n}-x^{*}\right\| \rightarrow 0$ as $n \rightarrow \infty$.

Proof Firstly, according to Lemma 2.7, the BVP (2) is equivalent to the integral formulation given by

$$
x(t)=\int_{0}^{1} H(t, s)[f(s, x(s), x(s))+g(s, x(s))] d s .
$$


Let $A_{2}: P \times P \rightarrow E$ be the operator defined by

$$
A_{2}(x, y)(t)=\int_{0}^{1} H(t, s) f(s, x(s), y(s)) d s
$$

and $B_{2}: P \rightarrow E$ be the operator defined by

$$
\left(B_{2} x\right)(t)=\int_{0}^{1} H(t, s) g(s, x(s)) d s .
$$

It is easy to prove that $x$ is the solution of the BVP (2) if and only if $x$ solves the operator equation $x=A_{2}(x, x)+B_{2} x$. Similar to the proof of Theorem 3.1, we can prove that $A_{2}$ : $P \times P \rightarrow P$ is a mixed monotone operator and satisfies $A_{2}\left(t x, t^{-1} y\right) \geq t^{\xi} A_{2}(x, y), B_{2}: P \rightarrow P$ is an increasing sub-homogeneous operator from $\left(\mathrm{H}_{1}\right),\left(\mathrm{H}_{2}\right)$, and Lemma 2.6. Secondly, we only need to prove that (i) and (ii) in Lemma 2.8. Next, we show that $A_{2}(h, h) \in P_{h}$ and $B_{2} h \in P_{h}$. We only need to verify the following conclusions:

(a) $\exists a_{1}^{\prime}>0$ and $a_{2}^{\prime}>0$, such that, for all $t \in[0,1], a_{2}^{\prime} h(t) \leq A_{2}(h, h)(t) \leq a_{1}^{\prime} h(t)$;

(b) $\exists b_{1}^{\prime}>0$ and $b_{2}^{\prime}>0$, such that, for all $t \in[0,1], b_{2}^{\prime} h(t) \leq B_{2} h(t) \leq b_{1}^{\prime} h(t)$.

Let

$$
\begin{aligned}
& a_{1}^{\prime}:=\frac{1}{\Gamma(\alpha)} \int_{0}^{1} \frac{(1-s)^{\alpha-1}}{1-\beta \eta^{\alpha-1}} f(s, 1,0) d s, \\
& a_{2}^{\prime}:=\frac{M_{0}}{\Gamma(\alpha)} \int_{0}^{1} \frac{s(1-s)^{\alpha-1}}{1-\beta \eta^{\alpha-1}} f(s, 0,1) d s, \\
& b_{1}^{\prime}:=\frac{1}{\Gamma(\alpha)} \int_{0}^{1} \frac{(1-s)^{\alpha-1}}{1-\beta \eta^{\alpha-1}} g(s, 1) d s, \\
& b_{2}^{\prime}:=\frac{M_{0}}{\Gamma(\alpha)} \int_{0}^{1} \frac{s(1-s)^{\alpha-1}}{1-\beta \eta^{\alpha-1}} g(s, 0) d s .
\end{aligned}
$$

It follows from $\left(\mathrm{H}_{1}\right),\left(\mathrm{H}_{2}\right)$, and Lemma 2.6 that

$$
\begin{aligned}
A_{2}(h, h)(t) & =\int_{0}^{1} H(t, s) f(s, h(s), h(s)) d s \\
& \leq \frac{1}{\Gamma(\alpha)} h(t) \int_{0}^{1} \frac{(1-s)^{\alpha-1}}{1-\beta \eta^{\alpha-1}} f(s, 1,0) d s \\
& =a_{1}^{\prime} h(t), \quad \forall t \in[0,1],
\end{aligned}
$$

and

$$
\begin{aligned}
A_{2}(h, h)(t) & =\int_{0}^{1} H(t, s) f(s, h(s), h(s)) d s \\
& \geq \frac{M_{0}}{\Gamma(\alpha)} h(t) \int_{0}^{1} \frac{s(1-s)^{\alpha-1}}{1-\beta \eta^{\alpha-1}} f(s, 0,1) d s \\
& =a_{2}^{\prime} h(t), \quad \forall t \in[0,1] .
\end{aligned}
$$

According to $\left(\mathrm{H}_{1}\right),\left(\mathrm{H}_{2}\right)$, and $\left(\mathrm{H}_{3}\right)$, we have

$$
f(s, 1,0) \geq f(s, 0,1) \geq \delta_{0} g(s, 0) \geq 0 .
$$


Since $g(t, 0) \not \equiv 0$, we obtain

$$
\int_{0}^{1} f(s, 1,0) d s \geq \int_{0}^{1} f(s, 0,1) d s \geq \delta_{0} \int_{0}^{1} g(s, 0) d s>0
$$

and, in consequence, $a_{1}^{\prime}>0$ and $a_{2}^{\prime}>0$. Therefore, $a_{2}^{\prime} h(t) \leq A_{2}(h, h)(t) \leq a_{1}^{\prime} h(t), t \in[0,1]$, and hence we get (a). In the same way, we get

$$
B_{2} h(t) \leq \frac{1}{\Gamma(\alpha)} h(t) \int_{0}^{1} \frac{(1-s)^{\alpha-1}}{1-\beta \eta^{\alpha-1}} g(s, 1) d s=b_{1}^{\prime} h(t)
$$

and

$$
B_{2} h(t) \geq \frac{M_{0}}{\Gamma(\alpha)} h(t) \int_{0}^{1} \frac{s(1-s)^{\alpha-1}}{1-\beta \eta^{\alpha-1}} g(s, 0) d s=b_{2}^{\prime} h(t)
$$

From $g(t, 0) \not \equiv 0$, we have $b_{1}^{\prime}>0$ and $b_{2}^{\prime}>0$. Therefore, $b_{2}^{\prime} h(t) \leq B_{2} h(t) \leq b_{1}^{\prime} h(t), t \in[0,1]$, and (b) is satisfied. Hence the condition (i) of Lemma 2.8 is proved. Next, we show that the condition (ii) of Lemma 2.8 is satisfied. For $x, y \in P$ and for any $t \in[0,1]$, according to $\left(\mathrm{H}_{3}\right)$, we have

$$
A_{2}(x, y)(t)=\int_{0}^{1} H(t, s) f(s, x(s), y(s)) d s \geq \delta_{0} \int_{0}^{1} H(t, s) g(s, u(s)) d s=\delta_{0} B_{2} u(t)
$$

Then we get $A_{2}(x, y) \geq \delta_{0} B_{2} x, x, y \in P$. Thus, the conclusions of Theorem 3.3 follow from Lemma 2.8.

From Lemma 2.9 we also prove the following theorem.

Theorem 3.4 Assume that $\left(\mathrm{H}_{1}\right),\left(\mathrm{H}_{2}\right),\left(\mathrm{H}_{5}\right)$, and $\left(\mathrm{H}_{6}\right)$ hold. Then

(1) there exist $x_{0}, y_{0} \in P_{h}$ and $\gamma \in(0,1)$ such that $\gamma y_{0} \leq x_{0}<y_{0}$ and

$$
\begin{aligned}
& x_{0}(t) \leq \int_{0}^{1} H(t, s)\left[f\left(t, x_{0}(s), y_{0}(s)\right)+g\left(s, x_{0}(s)\right)\right] d s, \quad t \in[0,1], \\
& y_{0}(t) \geq \int_{0}^{1} H(t, s)\left[f\left(s, y_{0}(s), x_{0}(s)\right)+g\left(s, x_{0}(s)\right)\right] d s, \quad t \in[0,1],
\end{aligned}
$$

where $h(t)=t^{\alpha-1}, t \in[0,1]$;

(2) the BVP (2) has a unique positive solution $x^{*}$ in $P_{h}$ and for any $x_{0}, y_{0} \in P_{h}$, constructing successively the sequences

$$
\begin{aligned}
& x_{n+1}(t)=\int_{0}^{1} H(t, s)\left[f\left(s, x_{n}(s), y_{n}(s)\right)+g\left(s, x_{n}(s)\right)\right] d s, \quad n=0,1,2, \ldots, \\
& y_{n+1}(t)=\int_{0}^{1} H(t, s)\left[f\left(s, y_{n}(s), x_{n}(s)\right)+g\left(s, y_{n}(s)\right)\right] d s, \quad n=0,1,2, \ldots
\end{aligned}
$$

we have $\left\|x_{n}-x^{*}\right\| \rightarrow 0$ and $\left\|y_{n}-x^{*}\right\| \rightarrow 0$ as $n \rightarrow \infty$.

Proof The proof is similar to that given for Theorem 3.3. We omit it. 


\section{Example}

In this section, we give two examples to illustrate our results.

Example 4.1 Consider the following boundary value problem:

$$
\left\{\begin{array}{l}
-D_{0+}^{\frac{5}{3}} x(t)=x^{\frac{1}{3}}+\arctan x+y^{-\frac{1}{3}}+t^{2}+t^{3}+\frac{\pi}{2}, \quad 0<t<1 \\
x(0)=x(1)=0
\end{array}\right.
$$

In this case, $\alpha=\frac{5}{3}$. Problem (14) can be regarded as a boundary value problem of the form (1) with $f(t, x, y)=x^{\frac{1}{3}}+y^{-\frac{1}{3}}+t^{2}+\frac{\pi}{2}$ and $g(t, x)=\arctan x+t^{3}$. Now we verify that conditions $\left(\mathrm{H}_{1}\right)-\left(\mathrm{H}_{4}\right)$ are satisfied.

Firstly, it is easy to see $\left(\mathrm{H}_{1}\right)$ and $\left(\mathrm{H}_{2}\right)$ are satisfied and $g(t, 0)=t^{3} \not \equiv 0$. Secondly, take $\delta_{0} \in(0,1]$, we obtain

$$
f(t, x, y)=x^{\frac{1}{3}}+y^{-\frac{1}{3}}+t^{2}+\frac{\pi}{2} \geq t^{2}+\frac{\pi}{2} \geq t^{3}+\arctan x \geq \delta_{0}\left(t^{3}+\arctan x\right)=\delta_{0} g(t, x) .
$$

Thus, $\left(\mathrm{H}_{3}\right)$ is satisfied. Moreover, for any $\lambda \in(0,1), t \in[0,1], x \in[0, \infty), y \in[0, \infty)$, we get $\arctan (\lambda x) \geq \lambda \arctan x$. Therefore

$$
\begin{aligned}
& g(t, \lambda x) \geq \lambda g(t, x) \\
& f\left(t, \lambda x, \lambda^{-1} y\right)=\lambda^{\frac{1}{3}} x^{\frac{1}{3}}+\lambda^{\frac{1}{3}} y^{-\frac{1}{3}}+t^{2}+\frac{\pi}{2} \geq \lambda^{\frac{1}{3}}\left(x^{\frac{1}{3}}+y^{-\frac{1}{3}}+t^{2}+\frac{\pi}{2}\right)=\lambda^{\gamma} f(t, x, y),
\end{aligned}
$$

where $\gamma=\frac{1}{3}$. We conclude that condition $\left(\mathrm{H}_{4}\right)$ is satisfied. Therefore, Theorem 3.1 ensures that the BVP (14) has a unique positive solution in $P_{h}$ with $h(t)=t^{\frac{1}{3}}(1-t)$.

Example 4.2 Consider the following boundary value problem:

$$
\left\{\begin{array}{l}
-D_{0+}^{\frac{3}{2}} x(t)=2 x^{\frac{1}{2}}+y^{-\frac{1}{2}}+t^{2}+t^{3}, \quad 0<t<1 \\
x(0)=0, \quad x(1)=\frac{1}{2} x\left(\frac{1}{2}\right)
\end{array}\right.
$$

In this case, $\alpha=\frac{3}{2}$. Problem (15) can be regard as a boundary value problem of form (2) with $f(t, x, y)=x^{\frac{1}{2}}+y^{-\frac{1}{2}}+t^{2}$ and $g(t, x)=x^{\frac{1}{2}}+t^{3}$. Now we verify that conditions $\left(\mathrm{H}_{1}\right)-\left(\mathrm{H}_{4}\right)$ are satisfied.

Firstly, it is easy to see $\left(\mathrm{H}_{1}\right)$ and $\left(\mathrm{H}_{2}\right)$ are satisfied and $g(t, 0)=t^{3} \not \equiv 0$. Secondly, take $\delta_{0} \in(0,1]$, we obtain

$$
f(t, x, y)=x^{\frac{1}{2}}+y^{-\frac{1}{2}}+t^{2} \geq x^{\frac{1}{2}}+t^{3} \geq \delta_{0}\left(x^{\frac{1}{2}}+t^{3}\right)=\delta_{0} g(t, x) .
$$

Thus, $\left(\mathrm{H}_{3}\right)$ is satisfied. Moreover, for any $\lambda \in(0,1), t \in[0,1], x \in[0, \infty), y \in[0, \infty)$, we have

$$
\begin{aligned}
& g(t, \lambda x)=\lambda^{\frac{1}{2}} x^{\frac{1}{2}}+t^{3} \geq \lambda^{\frac{1}{2}}\left(x^{\frac{1}{2}}+t^{3}\right) \geq \lambda g(t, x), \\
& f\left(t, \lambda x, \lambda^{-1} y\right)=\lambda^{\frac{1}{2}} x^{\frac{1}{2}}+\lambda^{\frac{1}{2}} y^{-\frac{1}{2}}+t^{2} \geq \lambda^{\frac{1}{2}}\left(x^{\frac{1}{2}}+y^{-\frac{1}{2}}+t^{2}\right)=\lambda^{\gamma} f(t, x, y),
\end{aligned}
$$

where $\gamma=\frac{1}{2}$. We conclude that condition $\left(\mathrm{H}_{4}\right)$ is satisfied. Therefore, Theorem 3.3 ensures that the BVP (15) has a unique positive solution in $P_{h}$ with $h(t)=t^{\frac{1}{2}}$. 


\section{Competing interests}

The authors declare that they have no competing interests.

\section{Authors' contributions}

All authors typed, read, and approved the final manuscript.

\section{Acknowledgements}

The authors would like to thank the referees for their valuable suggestions and comments.

Received: 3 November 2014 Accepted: 10 February 2015 Published online: 07 March 2015

\section{References}

1. Elsaid, A: Fractional differential transform method combined with the Adomian polynomials. Appl. Math. Comput. 218(12), 6889-6911 (2012)

2. Krasnosel'skii, MA: Positive Solutions of Operator Equations. Noordhoff, Groningen (1964)

3. Lakshmikantham, $V$, Vatsala, AS: General uniqueness and monotone iterative technique for fractional differential equations. Appl. Math. Lett. 21(8), 828-834 (2008)

4. Zhang, KM, Sun, JX: The relation between sign-changing solution and positive-negative solutions for nonlinear operator equations and its applications. Acta Math. Sci. 24(3), 463-468 (2004)

5. Zhang, KM: Nontrivial solutions of forth-order singular boundary value problems with sign-changing nonlinear terms. Topol. Methods Nonlinear Anal. 40(1), 53-70 (2012)

6. Sun, XB, Su, J, Han, MA: On the number of zeros Abelian integral for some Lienard systems of type $(4,3)$. Chaos Solitons Fractals 51, 1-12 (2013)

7. Rudin, W: Functional Analysis, 2nd edn. International Series in Pure and Applied Mathematics. McGraw-Hill, New York (1991)

8. Wei, ZL: Positive solution of singular Dirichlet boundary value problems for second order differential equation system. J. Math. Anal. Appl. 328(2), 1255-1267 (2007)

9. Zhang, XG, Liu, LS, Wu, YH: Positive solutions of nonresonance semipositone singular Dirichlet boundary value problems. Nonlinear Anal. TMA 68(1), 97-108 (2008)

10. Zhang, XG, Liu, LS, Wu, YH: The uniqueness of positive solutions for a fractional order model of turbulent flow in a porous medium. Appl. Math. Lett. 37, 26-33 (2014)

11. Lu, HX, Sun, L, Sun, JX: Existence of positive solutions to a non-positive elastic beam equation with both ends fixed. Bound. Value Probl. (2012). doi:10.1186/1687-2770-292-56

12. Zhang, $X Q$, Sun, JX: On multiple sign-changing solutions for some second-order integral boundary value problems. Electron. J. Qual. Theory Differ. Equ. 2010, 44 (2010)

13. Liu, YS: Positive solutions of boundary value problems for nonlinear singular differential equations in Banach spaces. Acta Math. Sin. 47(1), 131-140 (2004)

14. Lin, $X \mathrm{~L}$, Zhao, ZQ: Sign-changing solution for a third-order boundary value problem in ordered Banach space with lattice structure. Bound. Value Probl. 2014, 132 (2014). doi:10.1186/1687-2770-2014-132

15. Lin, $\mathrm{XL}$, Zhao, ZQ: Existence and uniqueness of symmetric positive solutions of $2 \mathrm{n}$-order nonlinear singular boundary value problems. Appl. Math. Lett. 26(7), 692-698 (2013)

16. Liu, RK, Ma, RY: Existence of positive solutions for an elastic beam equation with nonlinear boundary conditions. J. Appl. Math. 2014, 972135 (2014)

17. Dai, GW, Ma, RY: Bifurcation from intervals for Sturm-Liouville problems and its applications. Electron. J. Differ. Equ. 2014, 3 (2014)

18. Cheng, ZB: Existence of positive periodic solutions for third-order differential equations with strong singularity. Adv. Differ. Equ. (2014). doi:10.1186/1687-1847-2014-162

19. Cheng, ZB, Ren, JL: Some results for high-order generalized neutral differential equations. Adv. Differ. Equ. (2013). doi:10.11.186/1687-1847-2013-202

20. Zhao, XK, Ge, WG: Unbounded solutions for a fractional boundary value problem on the infinite interval. Acta Appl. Math. 109, 495-505 (2010)

21. Zhang, ZT: Existence of solutions for second order impulsive differential equations. Appl. Math. 12, 307-320 (1997)

22. Jiang, DQ, Yuan, CJ: The positive properties of the Green function for Dirichlet-type boundary value problems of nonlinear fractional differential equations and its application. Nonlinear Anal. TMA 72(2), 710-719 (2010)

23. Bai, ZB, Lü, HS: Positive solutions for boundary value problem of nonlinear fractional differential equation. J. Math. Anal. Appl. 311(2), 495-505 (2005)

24. Kosmatov, N: A singular boundary value problem for nonlinear differential equations of fractional order. J. Appl. Math Comput. (2008). doi:10.007/s12190-008-0104-X

25. Xu, XJ, Jiang, DQ, Hu, WM, O'Regan, D, Agarwal, PR: Positive properties of Green's function for three-point boundary value problems of nonlinear fractional differential equations and its applications. Appl. Anal. 91(2), 323-343 (2012)

26. Li, CF, Luo, XN, Zhou, Y: Existence of positive solutions of the boundary value problem for nonlinear fractional differential equations. Comput. Math. Appl. 59, 1363-1375 (2010)

27. Samko, SG, Kilbas, AA, Marichev, Ol: Fractional Integrals and Derivatives: Theory and Applications. Gordon \& Breach, Basel (1993)

28. Zhai, $\mathrm{CB}, \mathrm{Hoo}, \mathrm{M}$ : Fixed point theorem for mixed monotone operators with perturbation and applications to fractional differential equation boundary value problems. Nonlinear Anal. TMA 75, 2542-2551 (2012)

29. Zhai, CB, Yan, WP, Yang, C: A sum operator method for the existence and uniqueness of positive solution to Riemann-Liouville fractional differential equation boundary value problems. Commun. Nonlinear Sci. Numer. Simul. $18,858-866(2013)$ 